PROCEEDINGS OF THE

AMERICAN MATHEMATICAL SOCIETY

Volume 130, Number 3, Pages 769-772

S 0002-9939(01)06177-9

Article electronically published on August 29, 2001

\title{
INVERTIBILITY PRESERVING LINEAR MAPS AND ALGEBRAIC REFLEXIVITY OF ELEMENTARY OPERATORS OF LENGTH ONE
}

\author{
PETER ŠEMRL
}

(Communicated by David R. Larson)

\begin{abstract}
Let $X$ and $Y$ be real or complex Banach spaces. We show that a surjective linear map $\phi: \mathcal{B}(X) \rightarrow \mathcal{B}(Y)$ preserving invertibility in both directions is either of the form $\phi(T)=A T B$ or the form $\phi(T)=C T^{\prime} D$, where $A: X \rightarrow Y, B: Y \rightarrow X, C: X^{\prime} \rightarrow Y$, and $D: Y \rightarrow X^{\prime}$ are bounded invertible linear operators. As an application we improve a result of Larson and Sourour on algebraic reflexivity of elementary operators of length one.
\end{abstract}

\section{InTRODUCTION AND STATEMENT OF RESUlts}

In recent years a lot of work has been done on Kaplansky's problem [4 of characterizing linear maps preserving invertibility (see [1] for some historical remarks). The aim of this note is to provide a simple proof of a result of Jafarian and Sourour [3] on linear invertibility preserving maps acting between operator algebras $\mathcal{B}(X)$ and $\mathcal{B}(Y)$. Another advantage of this short proof is that it works not only in the complex case but also in the real case.

Let $X$ and $Y$ be real or complex Banach spaces. We denote by $\mathcal{B}(X)$ the algebra of all bounded linear transformations on $X$. The dual space of a Banach space $X$ is denoted by $X^{\prime}$ and the adjoint of an operator $T$ is denoted by $T^{\prime}$. A linear map $\phi: \mathcal{B}(X) \rightarrow \mathcal{B}(Y)$ preserves invertibility in both directions if for every $T \in \mathcal{B}(X)$ the operator $\phi(T)$ is invertible if and only if $T$ is invertible.

Theorem 1.1. Let $X$ and $Y$ be real or complex Banach spaces and let $\phi: \mathcal{B}(X) \rightarrow$ $\mathcal{B}(Y)$ be a surjective linear map preserving invertibility in both directions. Then either $\phi(T)=A T B$ for every $T \in \mathcal{B}(X)$ or $\phi(T)=C T^{\prime} D$ for every $T \in \mathcal{B}(X)$, where $A: X \rightarrow Y, B: Y \rightarrow X, C: X^{\prime} \rightarrow Y$, and $D: Y \rightarrow X^{\prime}$ are bounded invertible linear operators.

In the complex case this result was substantially improved by Sourour [9] who showed that under the additional assumption of injectivity it is enough to assume that $\phi$ preserves invertibility in one direction only in order to get the same conclusion. We do not know whether the real analogue of this result holds true.

Received by the editors July 22, 1998 and, in revised form, September 14, 2000.

2000 Mathematics Subject Classification. Primary 47B49.

This research was supported in part by a grant from the Ministry of Science of Slovenia.

(C)2001 American Mathematical Society 
A linear transformation $\phi$ on $\mathcal{B}(X)$ is called an elementary operator of length one if $\phi(T)=A T B, T \in \mathcal{B}(X)$, for some $A, B \in \mathcal{B}(X)$. Using Theorem 1.1 we improve a result of Larson and Sourour [6] on algebraic reflexivity of elementary operators of length one. An interested reader can find more information on the concept of algebraic reflexivity in 5, 6. Note that in the following theorem the assumption of infinite dimensionality is indispensable. This is a simple consequence of the fact that every matrix is similar to its transpose [10].

Theorem 1.2. Let $X$ be an infinite dimensional real or complex Banach space and let $\phi: \mathcal{B}(X) \rightarrow \mathcal{B}(X)$ be a surjective linear map such that for every $T \in \mathcal{B}(X)$ there exist invertible linear operators $A_{T}$ and $B_{T}$ such that $\phi(T)=A_{T} T B_{T}$. Then $\phi(T)=A T B, T \in \mathcal{B}(X)$, for some invertible linear operators $A$ and $B \in \mathcal{B}(X)$.

\section{Proofs}

Let $X$ be a Banach space and $X^{\prime}$ its dual. For $x \in X$ and $f \in X^{\prime}$ we denote by $x \otimes f$ the rank one operator given by $(x \otimes f) z=f(z) x, z \in X$. Note that every rank one operator in $\mathcal{B}(X)$ can be written in this form. If $\lambda$ is a scalar, then $I-\lambda x \otimes f$ is invertible if and only if $\lambda f(x) \neq 1$.

Lemma 2.1. Let $A \in \mathcal{B}(X), A \neq 0$. The following conditions are equivalent:

- A has rank 1.

- If $B \in \mathcal{B}(X)$ is invertible, then $B+\lambda A$ is invertible for all but at most one scalar $\lambda$.

Proof. Assume first that $B$ is invertible and $A=x \otimes f$ of rank one. Then $B+\lambda A=$ $B\left(I+\lambda B^{-1} A\right)$ is invertible if and only if $I+\lambda\left(B^{-1} x\right) \otimes f$ is invertible. According to the remark before the lemma $B+\lambda A$ is invertible for all but at most one scalar $\lambda$.

Assume now that a nonzero $A$ is not of rank one. Then there are vectors $x, y, u, v$ such that $A x=u$ and $A y=v$ are linearly independent. We can find an invertible operator $B$ such that $B x=-u$ and $B y=v$. Consequently, both $B+A$ and $B-A$ are singular. This completes the proof.

Lemma 2.2. Let $S, T \in \mathcal{B}(X)$ be invertible operators. Assume that for every rank one operator $R \in \mathcal{B}(X)$ the operator $S+R$ is invertible if and only if $T+R$ is invertible. Then $S=T$.

Proof. Assume first that there exists $x \in X$ such that $S^{-1} T x$ and $x$ are linearly independent. Then we can find a nilpotent $R$ of rank one satisfying $R x=-S^{-1} T x$. Then $S^{-1} T+R$ is not invertible, and consequently, $T+S R$ is not invertible. According to our assumption $S+S R$ is not invertible. The same must be true for $I+R$. This contradiction shows that $S^{-1} T x$ and $x$ are linearly dependent for every $x \in X$. Hence, $T=\lambda S$ for some nonzero scalar $\lambda$. It follows that for every $R$ of rank one $I+S^{-1} R$ is invertible if and only if $I+(\lambda S)^{-1} R$ is invertible, and consequently, $\lambda=1$. This completes the proof.

Proof of Theorem 1.1. First we will show that $\phi$ is injective. Assume to the contrary that there exist $T \in \mathcal{B}(X)$ and $x, u \in X$ such that $\phi(T)=0$ and $T x=u \neq 0$. Then we can find an invertible $S \in \mathcal{B}(X)$ satisfying $S x=u$. Consequently, $S-T$ is singular. Therefore $\phi(S-T)=\phi(S)$ is not invertible. This contradiction shows that $\phi$ is bijective. 
As already mentioned in the Introduction there is no loss of generality in assuming that $\phi(I)=I$. According to Lemma $2.1 \phi(T)$ is of rank one if and only if $T$ is of rank one. By a standard argument (see [2, 3, 9]) there exist either linear bijective transformations $E: X \rightarrow Y$ and $F: X^{\prime} \rightarrow Y^{\prime}$ such that $\phi(x \otimes f)=E x \otimes F f$, $x \in X, f \in X^{\prime}$, or linear bijective transformations $G: X^{\prime} \rightarrow Y$ and $H: X \rightarrow Y^{\prime}$ such that $\phi(x \otimes f)=G f \otimes H x, x \in X, f \in X^{\prime}$. In the first case $I-x \otimes f$ is invertible if and only if $\phi(I-x \otimes f)=I-E x \otimes F f$ is invertible, $x \in X, f \in X^{\prime}$. Hence, $f(x)=(F f)(E x)$. By the closed graph theorem $E$ and $F$ are continuous. Moreover, $F=\left(E^{-1}\right)^{\prime}$, and consequently, $\phi(T)=E T E^{-1}$ for every finite rank operator. Replacing $\phi$ by $T \mapsto E^{-1} \phi(T) E$ we can assume that $\phi(T)=T$ for every finite rank operator $T$. Similarly we treat the second case.

So, for every invertible $T \in \mathcal{B}(X)$ and rank one $R \in \mathcal{B}(X)$ we have that $T+R$ is invertible if and only if $\phi(T+R)=\phi(T)+R$ is invertible. Lemma 2.2 yields that $\phi(T)=T$ for every invertible $T$. Let $T \in \mathcal{B}(X)$ now be an arbitrary operator. If a scalar $\lambda$ is large enough, then $T-\lambda$ is invertible, and hence

$$
\phi(T)=\phi(T-\lambda)+\lambda=T .
$$

This completes the proof.

Proof of Theorem 1.2. As $\phi$ preserves invertibility in both directions it is either of the form $\phi(T)=A T B$ or of the form $\phi(T)=C T^{\prime} D$, where $A, B: X \rightarrow X$, $C: X^{\prime} \rightarrow X$, and $D: X \rightarrow X^{\prime}$ are bounded invertible linear operators. We have to show that the second possibility cannot occur. In this case every bounded linear operator on $X^{\prime}$ is an adjoint of an operator on $X$. This implies that $X$ is reflexive. It follows that there exists a separable subspace $W \subset X$ and a linear projection $P$ from $X$ onto $W$ such that $\|P\|=1$ [7. Proposition 1]. According to [8], there are sequences $\left(x_{n}\right) \in W$ and $\left(f_{n}\right) \in W^{\prime}$ such that the linear span of $\left(x_{n}\right)$ is dense in $W, f_{m}\left(x_{n}\right)=\delta_{m, n}, \sup _{n}\left\|x_{n}\right\|\left\|f_{n}\right\|<\infty$, and $x \in W$ and $f_{n}(x)=0$ for all positive integers $n$ imply $x=0$. For every positive integer $n$ we define a functional $g_{n} \in X^{\prime}$ by $g_{n}(x)=f_{n}(P x), x \in X$. The linear operator $T=I-P+\sum_{n=1}^{\infty} 2^{-n} x_{n+1} \otimes g_{n}$ is injective, but $T^{\prime} g_{1}=0$, and so the second form is not possible. This completes the proof.

\section{REFERENCES}

[1] B. Aupetit, Propriétés spectrales des algèbres de Banach, Lecture Notes in Mathematics 735, Springer-Verlag, 1979. MR 81i:46055

[2] M. Brešar, P. Šemrl, Linear preservers on $\mathcal{B}(X)$, Banach Center Publications 38 (1997), 49-58. MR 99c: 47044

[3] A.A. Jafarian, A.R. Sourour, Spectrum-preserving linear maps, J. Funct. Anal. 66 (1986), 255-261. MR 87 m:47011

[4] I. Kaplansky, Algebraic and analytic aspects of operator algebras, American Mathematical Society, Providence, 1970. MR 47:845

[5] D. Larson, Reflexivity, algebraic reflexivity, and linear interpolation, Amer. J. Math. 110 (1988), 283-299. MR 89d:47096

[6] D. Larson, A.R. Sourour, Local derivations and local automorphisms of $\mathcal{B}(X)$, Proc. Symp. Pure Math. 51 (1990), 187-194. MR 91k:47106

[7] J. Lindenstrauss, On nonseparable reflexive Banach spaces, Bull. Amer. Math. Soc. 72 (1966), 967-970. MR 34:4875

[8] R.I. Ovsepian, A. Pelczyński, Existence of a fundamental total and bounded biorthogonal sequence, Studia Math. 54 (1975), 149-159. MR 52:14942 
[9] A.R. Sourour, Invertibility preserving linear maps on $\mathcal{L}(X)$, Trans. Amer. Math. Soc. 348 (1996), 13-30. MR 96f:47069

[10] O. Taussky, H. Zassenhaus, On the similarity transformation between a matrix and its transpose, Pacific J. Math. 9 (1959), 893-896. MR 21:7216

Institute of Mathematics, Physics, and Mechanics, Jadranska 19, 1000 Ljubljana, SLOVENIA

E-mail address: peter.semrl@fmf.uni-lj.si 